\title{
Ciprofloxacin-Induced Acute Psychosis: A Case Report
}

\author{
Cilia Nazef*, Aazim Arif \\ Florida State University College of Medicine, Tallahassee Fl, United States of America \\ *Corresponding author: cnazef@gmail.com \\ Received May 01, 2019; Revised June 04, 2019; Accepted June 27, 2019
}

\begin{abstract}
Background: Ciprofloxacin, a member of the fluoroquinolone class, is a commonly used antimicrobial agent for treatment of both gram positive and gram negative bacterial infections. More commonly encountered adverse effects of ciprofloxacin relate to the gastrointestinal tract and include nausea, diarrhea, abnormal liver enzymes, and risk for Clostridium difficile infection. Other adverse effects include QT prolongation, dysglycemia, and risk for tendon rupture. It has been reported that minor CNS effects, such as dizziness and headaches, can result from the use of ciprofloxacin; however there have been rare occurrences of seizures and hallucinations [3,4]. Case Presentation: A 24 year old male patient presented to the primary care clinic with history of voluminous, watery diarrhea, fever, weight loss and abdominal pain for 10 days and has no significant past medical history. Workup demonstrated a positive fecal occult test and multiple pathogens in stool PCR. He was subsequently diagnosed with polymicrobial infectious gastroenteritis and colitis and was prescribed ciprofloxacin $500 \mathrm{mg}$ twice daily and metronidazole $500 \mathrm{mg}$ twice daily for 10 days. Four days later following antibiotics use, the patient had visited the clinic with complaints of feeling unwell, auditory hallucinations, and suicidal ideations without intent or plan. $\mathrm{He}$ was suspected to have ciprofloxacin induced psychosis and was instructed to discontinue ciprofloxacin but to continue taking metronidazole. After a week, the patient reported that his psychiatric symptoms had resolved two days after discontinuing ciprofloxacin. Conclusion: Ciprofloxacin-induced psychosis, albeit uncommon, should be included in the differential diagnosis if a patient begins to show signs and symptoms of psychosis during treatment with ciprofloxacin.
\end{abstract}

Keywords: ciprofloxacin, fluoroquinolones, psychosis

Cite This Article: Cilia Nazef, and Aazim Arif, "Ciprofloxacin-Induced Acute Psychosis: A Case Report." American Journal of Medical Case Reports, vol. 7, no. 7 (2019): 143-144. doi: 10.12691/ajmcr-7-7-6.

\section{Background}

Ciprofloxacin is a commonly used antimicrobial agent for treatment of both gram positive and gram negative bacterial infections. It belongs to class of drugs known as fluoroquinolones, and unlike other members of the class, ciprofloxacin is a non-respiratory agent. Fluoroquinolones act by inhibiting bacterial DNA gyrase and topoisomerase IV, two enzymes necessary for successful DNA replication, thus causing bactericidal action [1].

More commonly encountered adverse effects of ciprofloxacin relate to the gastrointestinal tract and include nausea, diarrhea, abnormal liver enzymes, and risk for Clostridium difficile infection. Other potential side effects, along with other members of the fluoroquinolone class, include QT interval prolongation, dysglycemia, rash, retinal detachment, risk of tendinopathy and tendon rupture, and the recently identified association of risk for aortic aneurysm rupture [2]. It has been reported that minor CNS effects, such as dizziness and headaches, can result from the use of ciprofloxacin; however there have been rare occurrences of seizures and hallucinations [3,4]. Several published reports of ciprofloxacin and levofloxacin induced psychosis and demonstrated that cessation of the medication resulted in resolution of the psychotic symptoms. However, the mechanism by which fluoroquinolones, in particular, ciprofloxacin, induces psychosis has not yet been fully understood [5].

It has been proposed that fluoroquinolones may inhibit the $\mathrm{GABA}_{\mathrm{A}}$ receptor, leading to an upregulation of glutamate. This mechanism is believed to play a role in the induction of seizures with fluoroquinolone use, but it is unclear if it also plays a role in the psychosis. [6] It is theorized that an increase in glutamate may subsequently lead to elevated AMPA receptor activation and manifestation of psychosis [7]. Furthermore, the question of the susceptibility to psychosis with ciprofloxacin with those who have a family history of similar adverse effects has not been studied.

\section{Case Presentation}

A 24 year old male patient presented to the primary care clinic with history of voluminous, watery diarrhea, fever, weight loss and abdominal pain for 10 days and has no significant past medical history. He informed that he had recently returned after three weeks from a trip to India, where he was fairly conscious of food choices and abstained from drinking local water; however, he may 
have had exposure to the local drinking water. In the clinic, on examination, diffuse tenderness on palpation and hyperactive bowel sounds were observed and his vitals were stable. Further workup demonstrated a positive fecal occult blood test and gastrointestinal polymerase chain reaction presence of Campylobacter jejuni, C. coli, C. upsaliensis, Plesiomonas shigelloides, Salmonella enterica, Enteroaggregative E. coli, Enteropathogenic $E$. coli, and Enterotoxigenic E. coli. He was subsequently diagnosed with polymicrobial infectious gastroenteritis and colitis and was prescribed ciprofloxacin $500 \mathrm{mg}$ twice daily and metronidazole $500 \mathrm{mg}$ twice daily for 10 days.

Four days later following antibiotics use, the patient had visited the clinic with complaints of feeling unwell, auditory hallucinations, and suicidal ideations without intent or plan. He denied past psychiatric history, psychiatric family history, and illicit drug use. Upon further questioning, patient stated that he has had family members, including his paternal grandmother and uncle, who had similar neurocognitive responses when taking ciprofloxacin. On examination, he was in mild distress with regard to his psychiatric symptoms. He was suspected to have ciprofloxacin induced psychosis and was instructed to discontinue ciprofloxacin but to continue taking metronidazole. He was advised to follow up a week later if the symptoms did not progress. After a week, the patient reported that his psychiatric symptoms had resolved two days after discontinuing ciprofloxacin. Further, his diarrhea had resolved to two soft bowel movements per day.

\section{Discussion}

The patient developed acute psychosis due to ciprofloxacin used for treatment of polymicrobial gastroenteritis and this finding is supported by earlier reports of fluoroquinolone induced psychosis. The mechanism by which ciprofloxacin induces psychosis is unclear at the moment. A proposed theory is that ciprofloxacin and other fluoroquinolones may inhibit GABA at its receptor and lead to CNS excitation.
Age is typically taken into consideration when a patient presents with symptoms of psychosis, thus this adverse effects is important to recognize in a younger population as these symptoms may be confused for a primary psychiatric disorder. It is also beneficial to note that with cessation of the medication, the psychosis resolves. Further, the patient in this case has family members who had similar reactions to ciprofloxacin, which may point to a familial component of this specific adverse reaction.

\section{Conclusion}

Ciprofloxacin-induced psychosis, albeit uncommon, should be included in the differential diagnosis if a patient begins to show signs and symptoms of psychosis during treatment with ciprofloxacin. Further, there may be a genetic component, and if so, it may be advisable to avoid ciprofloxacin in a patient who has a family history of ciprofloxacin-induced psychosis

\section{References}

[1] Hooper DC. Mechanisms of action of antimicrobials: focus on fluoroquinolones. Clin Infect Dis. (2001); 32(Suppl 1): S9-S15.

[2] Pasternak B, Inghammar M, Svanström H. Fluoroquinolone use and risk of aortic aneurysm and dissection: nationwide cohort study. BMJ. (2018); 360: k678. Published 2018 Mar 8.

[3] Carbon, C. Comparison of side effects of levogloxacin versus other lfuorquionolones. Chemotherpay. (2001); 47 (s3): 9-14.

[4] LeBel, M. Ciprofloxacin: chemistry, mechanism of action, resistance, antimicrobial apectrum, pharmacokinetics, clinical trials, and adverse reactions. Pharmacotherapy. (1988); 8, 3-30.

[5] Ranjan, A., Praharaj, S. K. Ciprofloxacin-induced psychosis J Neuropsychiatry Clin Neurosci. (2014); 26(1), E36-37.

[6] Segev S, Rehavi M, Rubinstein E. Quinolones, theophylline, and diclofenac interactions with the gamma-aminobutyric acid receptor. Antimicrob Agents Chemother. 1988; 32(11): 1624-1626.

[7] Kalkman, H. \& Loetscher, E. GAD67: the link between the GABA-deficit hypothesis and the daminergic- and glutamatergic theories of psychosis. J Neural Transm (2003) 110: 803 